A NNALES

UNIVERSITATIS MARIAE CURIE-SKŁODOWSKA

L UBLIN - P OLONIA

VOL. LXVIII, NO. 2, 2014

SECTIO A

$19-26$

WALDEMAR CIEŚLAK, HORST MARTINI and WITOLD MOZGAWA

\title{
Rotation indices related to Poncelet's closure theorem
}

\begin{abstract}
Let $C_{R} C_{r}$ denote an annulus formed by two non-concentric circles $C_{R}, C_{r}$ in the Euclidean plane. We prove that if Poncelet's closure theorem holds for $k$-gons circuminscribed to $C_{R} C_{r}$, then there exist circles inside this annulus which satisfy Poncelet's closure theorem together with $C_{r}$, with $n$ gons for any $n>k$.
\end{abstract}

1. Introduction. Poncelet's closure theorem, going back to the 19th century, has various interesting forms and applications; cf. [2], [7], [4], [9], and the excellent survey [3] as well as [4]. The rich history of this theorem is presented in [1, Ch. 16], [8, § 2.4], and [7], and our paper refers to circular versions of it. Let $C_{R}, C_{r}$ be two circles with radii $R>r>0$ and $C_{r}$ lying inside $C_{R}$. From any point on $C_{R}$, draw a tangent to $C_{r}$ and extend it to $C_{R}$ again, using the obtained new intersection point with $C_{R}$ for starting with a new tangent to $C_{r}$, etc.; the system of tangential segments obtained in this way inside $C_{R}$ is called a Poncelet transverse (or bar billiard). We say that the annulus $C_{R} C_{r}$ has Poncelet's porism property if there is a starting point on $C_{R}$ for which a Poncelet traverse is a closed polygon. Poncelet's closure theorem (for circles) says that then the transverse will also close for any other starting point from $C_{R}$. It is known that such closing polygons (with or without self-intersections) correspond to rational rotations; e.g.,

2010 Mathematics Subject Classification. 51M04, 51N20, 52A10, 53A04.

Key words and phrases. Bar billiards, Euler's triangle formula, Poncelet's closure theorem, Poncelet's porism property. 
the rotation number or index $\frac{1}{3}$ is related to a triangle "between" $C_{R}$ and $C_{r}$, and the index $\frac{2}{5}$ to a (self-intersecting) pentagram.

In [6] it was proved that "close" to a pair of circles, which have Poncelet's porism property for index $\frac{1}{3}$, there exist unique pairs of circles having this property with respect to indices $\frac{1}{4}$ and $\frac{1}{6}$, and it was conjectured there that this holds true for arbitrary indices.

In the present paper we show that this conjecture is true in the following sense: for a pair of circles having Poncelet's porism property for index $\frac{1}{k}$, with $k \geq 3$ as natural number, we prove that there exists a circle lying between the starting circles such that this circle together with the smaller given circle has Poncelet's porism property for any given index $\frac{1}{n}$, where $n$ is an arbitrary natural number with $n>k$.

2. Basic notions and tools. Let us consider a circular annulus $C_{r} C_{a, R}$ formed by two circles $C_{r}$ and $C_{a, R}$. The circles $C_{r}$ and $C_{a, R}$ are given by the equations $x^{2}+y^{2}=r^{2}$ and $(x-a)^{2}+y^{2}=R^{2}$, respectively, with

$$
0<a<R-r \text {. }
$$

Recall the following form of Poncelet's closure theorem which is suitable for our purpose; see [1].

If there exists a one circuminscribed (i.e., simultaneously inscribed in the outer circle and circumscribed about the inner circle) $n$-gon in a circular annulus, then any point of the outer circle is the vertex of some circuminscribed n-gon.

If Poncelet's closure theorem holds for $n=3$, then Euler's condition

$$
R^{2}-2 R r-a^{2}=0
$$

is satisfied. We will denote this condition by $\operatorname{Pct}\left(C_{r} C_{a, R}, 3\right)$. There is no elementary formula for the analogously defined condition Pct $\left(C_{r} C_{a, R}, n\right)$, but we note that $\operatorname{Pct}\left(C_{r} C_{a, R}, 4\right)$ and $\operatorname{Pct}\left(C_{r} C_{a, R}, 6\right)$ have the forms

$$
\left(R^{2}-a^{2}\right)^{2}=2 r^{2}\left(R^{2}+a^{2}\right)
$$

and

$$
3\left(R^{2}-a^{2}\right)^{4}=4 r^{2}\left(R^{2}+a^{2}\right)\left(R^{2}-a^{2}\right)^{2}+16 r^{2} a^{2} R^{2},
$$

respectively; see [3].

It is amazing that for particular natural numbers we have elementary conditions involving also radicals, while for an arbitrary natural number $n \geq$ 3 only the Jacobi formula (cf. formula (7) in [10]), using elliptic functions, is involved.

For further use we introduce a convenient parametrization of the annulus $C_{r} C_{a, R}$. Namely, we take the parametrization $z(t)=r e^{i t}$ for $C_{r}$, and for $C_{a, R}$ we use

$$
w(t)=z(t)+\lambda(t) i e^{i t}, \quad t \in[0,2 \pi],
$$


where $\lambda(t)=\sqrt{R^{2}-(r-a \cos t)^{2}}-a \sin t$.

The line which is tangent to the circle $C_{r}$ at a point $z(t)$ intersects the circle $C_{R}$ at a point $w(t)=z(t)+\lambda(t) i e^{i t}$. Let us draw a second tangent line to $C_{r}$, passing at $w(t)$. It intersects $C_{r}$ at a point $z(\varphi(t))$, where $\varphi(t)$ satisfies the condition

$$
\tan \frac{\varphi(t)-t}{2}=\frac{\lambda(t)}{r} .
$$

In [5] it is proved that

$$
\varphi^{\prime}=\frac{\sqrt{1-(\sigma \circ \varphi)^{2}}}{\sqrt{1-\sigma^{2}}},
$$

where

$$
\sigma(t)=\frac{r-a \cos t}{R} .
$$

It is routine to check that the solution of this differential equation with initial condition $\varphi(0)=m$ is given by the formula

$$
\varphi(t)=B^{-1}(B(t)+B(m)),
$$

where

$$
B(t)=\int_{0}^{t} \frac{d s}{\sqrt{1-\sigma^{2}(s)}} .
$$

\section{Results and proofs.}

Theorem 1. Poncelet's closure theorem holds in the annulus $C_{r} C_{a, R}$ for $n$-gons, $n \geq 3$, if and only if the following identity holds:

$$
B\left(t+2 \arctan \frac{\lambda(t)}{r}\right) \equiv B(t)+\frac{1}{n} B(2 \pi) .
$$

Proof. $\Rightarrow)$ From the assumption it follows that Poncelet's transverse closes after $n$ reflections, forming a circuminscribed convex $n$-gon. This is equivalent to the condition

$$
\varphi^{[n]}(t)=t+2 \pi \quad \text { for all } t \in \mathbb{R},
$$

where

$$
\varphi^{[1]}=\varphi \quad \text { and } \quad \varphi^{[n+1]}=\varphi^{[n]} \circ \varphi \quad \text { for } n=1,2,3, \ldots
$$

Note that formula (9) implies

$$
\varphi^{[n]}(t)=B^{-1}(B(t)+n B(m)) .
$$

From (12) and (14) it follows immediately that

$$
B(2 \pi)=n B(m) .
$$


Finally, the function $\varphi$ is given by the formula

$$
\varphi(t)=B^{-1}\left(B(t)+\frac{1}{n} B(2 \pi)\right),
$$

and

$$
\varphi(0)=m=B^{-1}\left(\frac{1}{n} B(2 \pi)\right) .
$$

From (6) we get

$$
\varphi(t)=t+2 \arctan \frac{\lambda(t)}{r} .
$$

The formulas (17) and (18) imply the identity (11).

$\Leftarrow)$ Assume that in the annulus $C_{r} C_{a, R}$ the identity (11) holds for some natural number $n \geq 3$. From the formulas (10) and (16) we get

$$
\varphi^{[n]}(t)=B^{-1}(B(t)+B(2 \pi))=B^{-1}(B(t+2 \pi))=t+2 \pi .
$$

Now, using (10), we can rewrite the identity (11) in the form

$$
\int_{0}^{t+2 \arctan } \frac{1}{\sqrt{1-\sigma^{2}(s)}} d s \equiv \int_{0}^{t} \frac{1}{\sqrt{1-\sigma^{2}(s)}} d s+\frac{1}{n} \int_{0}^{2 \pi} \frac{1}{\sqrt{1-\sigma^{2}(s)}} d s .
$$

Hence we have

$$
\int_{t}^{2 \arctan } \frac{1}{\sqrt{1-\sigma^{2}(s)}} d s \equiv \frac{1}{n} \int_{0}^{2 \pi} \frac{1}{\sqrt{1-\sigma^{2}(s)}} d s .
$$

In the particular case $t=0$ we have

$$
\int_{0}^{2 \arctan \frac{1}{r} \sqrt{R^{2}-(r-a)^{2}}} \frac{1}{\sqrt{1-\sigma^{2}(s)}} d s=\frac{1}{n} \int_{0}^{2 \pi} \frac{1}{\sqrt{1-\sigma^{2}(s)}} d s .
$$

This is exactly the formula (5.6) from [5], and we note that it implies Poncelet's porism property for $n$-gons.

Introducing

$$
V_{\xi}=\frac{1}{r} \sqrt{[(1-\xi) r+\xi R]^{2}-(r-\xi a)^{2}}
$$

for $\xi \in[0,1]$, we have

$$
V_{\xi}=\frac{1}{r} \sqrt{(R-r+a)\left[(R-r-a) \xi^{2}+2 r \xi\right]} .
$$


Since $0<a<R-r$, we can write

$$
V_{\xi}=\frac{1}{r} c(\xi) \sqrt{R-r+a} \quad \text { for } \xi \in[0,1]
$$

where

$$
c(\xi)=\sqrt{(R-r-a) \xi^{2}+2 r \xi} .
$$

Note that

$$
V_{1}=\frac{1}{r} \sqrt{R^{2}-(r-a)^{2}} \quad \text { and } \quad V_{0}=0 .
$$

Similarly, we define

$$
\sigma_{\xi}(t)=\frac{r-\xi a \cos t}{(1-\xi) r+\xi R} \quad \text { for } \xi \in[0,1],
$$

and one has $\sigma_{1}=\sigma$ and $\sigma_{0}=1$.

Now we will prove our main theorem.

Theorem 2. Assume that Poncelet's closure theorem holds in an annulus $C_{r} C_{a, R}$ for $k$-gons, $k \geq 3$. Then for any $n>k$ there exists $\gamma \in(0,1)$ such that Poncelet's closure theorem holds in the annulus $C_{r} C_{\gamma a,(1-\gamma) r+\gamma R}$ for $n$-gons.

Proof. Using the equality (20) from the proof of Theorem 1, we introduce the function

$$
F_{n}(\xi)=n \int_{0}^{2 \arctan V_{\xi}} \frac{1}{\sqrt{1-\sigma_{\xi}^{2}(s)}} d s-\int_{0}^{2 \pi} \frac{1}{\sqrt{1-\sigma^{2}(s)}} d s .
$$

First we have

$$
F_{n}(1)=n \int_{0}^{2 \arctan V_{1}} \frac{1}{\sqrt{1-\sigma^{2}(s)}} d s-\int_{0}^{2 \pi} \frac{1}{\sqrt{1-\sigma^{2}(s)}} d s .
$$

From now on we assume that the starting annulus $C_{r} C_{a, R}$ has Poncelet's porism property for a natural number $k \geq 3$, and we consider $n>k$. Then by (20) we have

$$
k \int_{0}^{2 \arctan V_{1}} \frac{1}{\sqrt{1-\sigma^{2}(s)}} d s=\int_{0}^{2 \pi} \frac{1}{\sqrt{1-\sigma^{2}(s)}} d s .
$$


Using this condition, we get

$$
\begin{aligned}
F_{n}(1)= & (n-k) \int_{0}^{2 \arctan V_{1}} \frac{1}{\sqrt{1-\sigma^{2}(s)}} d s+k \int_{0}^{2 \arctan V_{1}} \frac{1}{\sqrt{1-\sigma^{2}(s)}} d s \\
& -\int_{0}^{2 \pi} \frac{1}{\sqrt{1-\sigma^{2}(s)}} d s=(n-k) \int_{0}^{2 \arctan V_{1}} \frac{1}{\sqrt{1-\sigma^{2}(s)}} d s>0 .
\end{aligned}
$$

In order to evaluate $F_{n}(0)$, we first calculate the value $F_{n}(\varepsilon)$ for $\varepsilon \in(0,1)$. We have

$$
\begin{aligned}
F_{n}(\varepsilon) & =n \int_{0}^{2 \arctan V_{\epsilon}} \frac{1}{\sqrt{1-\sigma_{\varepsilon}^{2}(s)}} d s-\int_{0}^{2 \pi} \frac{1}{\sqrt{1-\sigma_{\varepsilon}^{2}(s)}} d s \\
& =(n-1) \int_{0}^{2 \arctan V_{\epsilon}} \frac{1}{\sqrt{1-\sigma_{\varepsilon}^{2}(s)}} d s-\int_{2 \arctan V_{\epsilon}}^{2 \pi} \frac{1}{\sqrt{1-\sigma_{\varepsilon}^{2}(s)}} d s .
\end{aligned}
$$

First we prove that

$$
\lim _{\varepsilon \rightarrow 0^{+}} \int_{0}^{2 \arctan V_{\epsilon}} \frac{1}{\sqrt{1-\sigma_{\varepsilon}^{2}(s)}} d s \leq C,
$$

for some positive constant $C$. We calculate

$$
\begin{aligned}
& \int_{0}^{2 \arctan V_{\epsilon}} \frac{1}{\sqrt{1-\sigma_{\varepsilon}^{2}(s)}} d s \\
= & \int_{0}^{2 \arctan \frac{1}{r} c(\varepsilon) \sqrt{R-r+a}}\left[1-\left(\frac{r-a \varepsilon \cos t}{(1-\varepsilon) r+\varepsilon R}\right)^{2}\right]^{-\frac{1}{2}} d t \\
= & \int_{0}^{2 \arctan \frac{1}{r} c(\varepsilon) \sqrt{R-r+a}}\left(\frac{[(1-\varepsilon) r+\varepsilon R]^{2}-(r-\varepsilon a \cos t)^{2}}{((1-\varepsilon) r+\varepsilon R)^{2}}\right)^{-\frac{1}{2}} d t \\
= & \int_{0}^{2 \arctan \frac{1}{r} c(\varepsilon) \sqrt{R-r+a}} \frac{(1-\varepsilon) r+\varepsilon R}{\sqrt{(R-r+a \cos t)\left[(R-r-a \cos t) \varepsilon^{2}+2 r \varepsilon\right]}} d t
\end{aligned}
$$




$$
\begin{aligned}
& \leq \int_{0}^{2 \arctan \frac{1}{r} c(\varepsilon) \sqrt{R-r+a}} \frac{(1-\varepsilon) r+\varepsilon R}{\sqrt{(R-r-a)\left[(R-r-a) \varepsilon^{2}+2 r \varepsilon\right]}} d t \\
& =[(1-\varepsilon) r+\varepsilon R] \int_{0}^{2 \arctan \frac{1}{r} c(\varepsilon) \sqrt{R-r+a}} \frac{1}{c(\varepsilon) \sqrt{R-r-a}} d t \\
& =[(1-\varepsilon) r+\varepsilon R] \frac{2 \arctan \frac{1}{r} c(\varepsilon) \sqrt{R-r+a}}{c(\varepsilon) \sqrt{R-r+-a}} .
\end{aligned}
$$

Since $\arctan x<x$ for $x>0$, then

$$
\int_{0}^{2 \arctan V_{\epsilon}} \frac{1}{\sqrt{1-\sigma_{\varepsilon}^{2}(s)}} d s \leq \frac{2}{r}[(1-\varepsilon) r+\varepsilon R] \frac{\sqrt{R-r+a}}{\sqrt{R-r-a}} .
$$

Thus

$$
\lim _{\varepsilon \rightarrow 0^{+}} \int_{0}^{2 \arctan V_{\epsilon}} \frac{1}{\sqrt{1-\sigma_{\varepsilon}^{2}(s)}} d s \leq C=\frac{2}{r} \frac{\sqrt{R-r+a}}{\sqrt{R-r-a}} .
$$

Next, we claim that

$$
\lim _{\varepsilon \rightarrow 0^{+}} \int_{2 \arctan V_{\epsilon}}^{2 \pi} \frac{1}{\sqrt{1-\sigma_{\varepsilon}^{2}(s)}} d s=+\infty .
$$

We have

$$
\begin{aligned}
& \int_{2 \arctan V_{\epsilon}}^{2 \pi} \frac{1}{\sqrt{1-\sigma_{\varepsilon}^{2}(s)}} d s \\
& =\int_{2 \arctan V_{\epsilon}}^{2 \pi} \frac{(1-\varepsilon) r+\varepsilon R}{\sqrt{R-r+a \cos t} \cdot \sqrt{(R-r-a \cos t) \varepsilon^{2}+2 r \varepsilon}} d t
\end{aligned}
$$

and, furthermore,

$$
\begin{aligned}
& ((1-\varepsilon) r+\varepsilon R) \int_{2 \arctan V_{\epsilon}}^{2 \pi} \frac{1}{\sqrt{R-r+a} \cdot \sqrt{(R-r+a) \varepsilon^{2}+2 r \varepsilon}} d t \\
& =\frac{(1-\varepsilon) r+\varepsilon R}{\sqrt{R-r+a}} \cdot \frac{2 \pi-2 \arctan \frac{1}{r} \sqrt{R-r+a} \cdot c(\varepsilon)}{\sqrt{(R-r+a) \varepsilon^{2}+2 r \varepsilon}} \longrightarrow+\infty,
\end{aligned}
$$

when $\varepsilon \rightarrow 0$. Hence

$$
\lim _{\varepsilon \rightarrow 0^{+}} \int_{2 \arctan V_{\epsilon}}^{2 \pi} \frac{1}{\sqrt{1-\sigma_{\varepsilon}^{2}(s)}} d s=+\infty .
$$


Thus, we have

$$
F_{n}\left(0^{+}\right)=\lim _{\varepsilon \rightarrow 0^{+}} F_{n}(\varepsilon)=-\infty
$$

and

$$
F_{n}(1)>0 \text {. }
$$

These conditions imply that there exists a number $\gamma \in(0,1)$ such that

$$
F_{n}(\gamma)=0
$$

Thus, with Theorem 1 the proof is finished.

\section{REFERENCES}

[1] Berger, M., Geometry, I and II, Springer, Berlin, 1987.

[2] Black, W. L., Howland, H. C., Howland, B., A theorem about zigzags between two circles, Amer. Math. Monthly 81 (1974), 754-757.

[3] Bos, H. J. M., Kers, C., Dort, F., Raven, D. W., Poncelet's closure theorem, Expo. Math. 5 (1987), 289-364.

[4] Cima, A., Gasull, A., Manosa, V., On Poncelet's maps, Comput. Math. Appl. 60 (2010), 1457-1464.

[5] Cieślak, W., The Poncelet annuli, Beitr. Algebra Geom. 55 (2014), 301-309.

[6] Cieślak, W., Martini, H., Mozgawa, W., On the rotation index of bar billiards and Poncelet's porism, Bull. Belg. Math. Soc. Simon Stevin 20 (2013), 287-300.

[7] Lion, G., Variational aspects of Poncelet's theorem, Geom. Dedicata 52 (1994), 105118.

[8] Martini, H., Recent results in elementary geometry, Part II, Symposia Gaussiana, Proc. 2nd Gauss Symposium (Munich, 1993), de Gruyter, Berlin and New York, 1995, 419-443.

[9] Schwartz, R., The Poncelet grid, Adv. Geom. 7 (2007), 157-175.

[10] Weisstein, E. W., Poncelet's Porism, http:/mathworld. wolfram. com/Ponceletsporism.html

Waldemar Cieślak

Department of Applied Mathematics

Lublin University of Technology

ul. Nadbystrzycka 40

20-618 Lublin

Poland

Witold Mozgawa

Institute of Mathematics

Maria Curie-Skłodowska University

pl. M. Curie-Skłodowskiej 1

20-031 Lublin

Poland

e-mail: mozgawa@poczta.umcs.lublin.pl

Received November 6, 2013

\author{
Horst Martini \\ Faculty of Mathematics \\ Technical University Chemnitz \\ 09107 Chemnitz \\ Germany \\ e-mail: martini@mathematik.tu-chemnitz.de
}

